\title{
Student selected components as an educational platform for teaching medical students about clinical pharmacology and quality improvement activities
}

\author{
Olayinka A. Ogundipe*
}

Department of Medicine of the Elderly, Royal Infirmary of Edinburgh, Edinburgh, Scotland

Received: 28 December 2020

Revised: 13 February 2021

Accepted: 16 February 2021

\section{*Correspondence:}

Dr. Olayinka A. Ogundipe,

Email: ola_ayodele@hotmail.com

Copyright: $(9$ the author(s), publisher and licensee Medip Academy. This is an open-access article distributed under the terms of the Creative Commons Attribution Non-Commercial License, which permits unrestricted non-commercial use, distribution, and reproduction in any medium, provided the original work is properly cited.

\begin{abstract}
Student selected components (SSCs) are increasingly described elements of medical undergraduate education, training and curricula. SSCs offer the potential for integration into both traditional ('pre-clinical' versus 'clinical') medical curricula, as well as into other innovative or evolving medical training curricula. This article employs a structured and descriptive approach to exemplify the process by which year 1 medical students were supported in a practical manner to undertake a distinct small group SSC project. In this illustration, the SSC was focused on a quality improvement (QI) topic of relevance to clinical pharmacology and therapeutics (CPT), and involved a review of the anticholinergic burden of inpatient prescriptions for a defined cohort. The SSC was completed in the context of a teaching hospital's medicine of the elderly (MoE) clinical service. In a sequential manner, the paper describes experiential learning points from the perspective of a supervisor of an SSC project. The paper offers educational value with a potential for generalisable application to non-clinical and clinical educationalists. Furthermore, the paper offers guidance to supervisors, teachers, tutors and facilitators, with encouragement to consider how they may design similar projects for the training of undergraduate medical students in centres that they are affiliated with. The paper also highlights another key driver for productive SSCs i.e. the central principle of striving to promote projects and activities that support active student engagement, rather than merely passive inclusion.
\end{abstract}

Keywords: Anticholinergic burden, Clinical pharmacology, Medical education, Medicine of the elderly, Student selected component, Quality improvement

\section{INTRODUCTION}

Student selected components (SSCs) (or equivalent activities) are increasingly described elements of undergraduate medical curricula and training. ${ }^{1}$ One general aim is that SSCs strive to accommodate students' choices within the framework of otherwise fixed elements of medical training. Beyond that, the specific aims of SSCs vary across undergraduate medical programmes, while striving to retain the major learning objectives. ${ }^{1,2}$
Dependent upon an individual institution's ability to optimally plan, structure, resource (e.g. adequate time and personnel), deliver, monitor, and review SSC programmes, there is scope for attaining comparable learning outcomes across different hosting departments (e.g. within the same institution), and also potentially across diverse medical schools. ${ }^{1,2}$ For the latter outcome to be achieved and maintained, SSC programmes require an essential driver i.e. ensuring that the delivery of activities support an approach of active student engagement, rather than passive inclusion. ${ }^{3.4}$ 
A practical challenge is the need to optimally train supervisors/teachers/tutors/facilitators (hereafter referred to as STTFs) to have a clear idea of the remit and goals of SSCs, and also on their need to gain an understanding of how they may best support SSCs. Another challenge is the need to ensure that medical students acquire an early and clear understanding of how they may best actively engage with SSCs in a manner that is planned, purposeful and outcome-pursuant. ${ }^{3.4}$

Previous authors have published work describing 12 tips for developing and sustaining SSC programmes. ${ }^{5}$ This author group also described tips for how medical students could better prepare themselves towards getting the most out of SSC projects. ${ }^{6}$

Using the 12 tips structured approach and examples, this write-up describes the process by which year $1 \mathrm{MBChB}$ students were practically supported in undertaking a defined SSC project. In this instance, the SSC focused on a specified quality improvement (QI) topic related to clinical pharmacology and therapeutics (CPT), and was undertaken in the context of a teaching hospital's clinical service.

\section{METHODS}

The focus of this paper is both descriptive (of the process used), and educational (to highlight the potential educational value to be derived) using an illustrated SSC, rather than a narrative emphasis on the actual results of the QI project.

This paper describes a low fidelity undergraduate medical SSC project focused on QI activities (QIA) on a topic of relevance to $\mathrm{CPT}$. The paper draws heavily upon the earlier cited 12 tips approach and other previously published works on student training and/or SSCs. ${ }^{5-11}$ The aforementioned were used as both a foundation and framework to describe the application to developing this SSC.

Using each of the 12 tips as a descriptive sub-heading, the paper practically exemplifies how the SSC was actualised in a staged manner. ${ }^{5,6}$ To enhance the practical illustration, this descriptive report is further sub-divided into three phases of: 'before', 'during,' and 'after' the SSC (Figure $1)$.

Each tip is then framed predominantly as action phrases or verbs, to emphasise the need for proactivity among STTFs and students alike. In addition, paragraphs are incorporated to more readily delineate salient points within each tip.

The broad aim of this SSC was to support 'early-year' students (year $1 \mathrm{MBChB}$ in the Edinburgh Medical School; a six-year intercalated degree curriculum) in undertaking a consultant designed, and postgraduate doctor supervised small group project. Six students were assigned to this SSC.

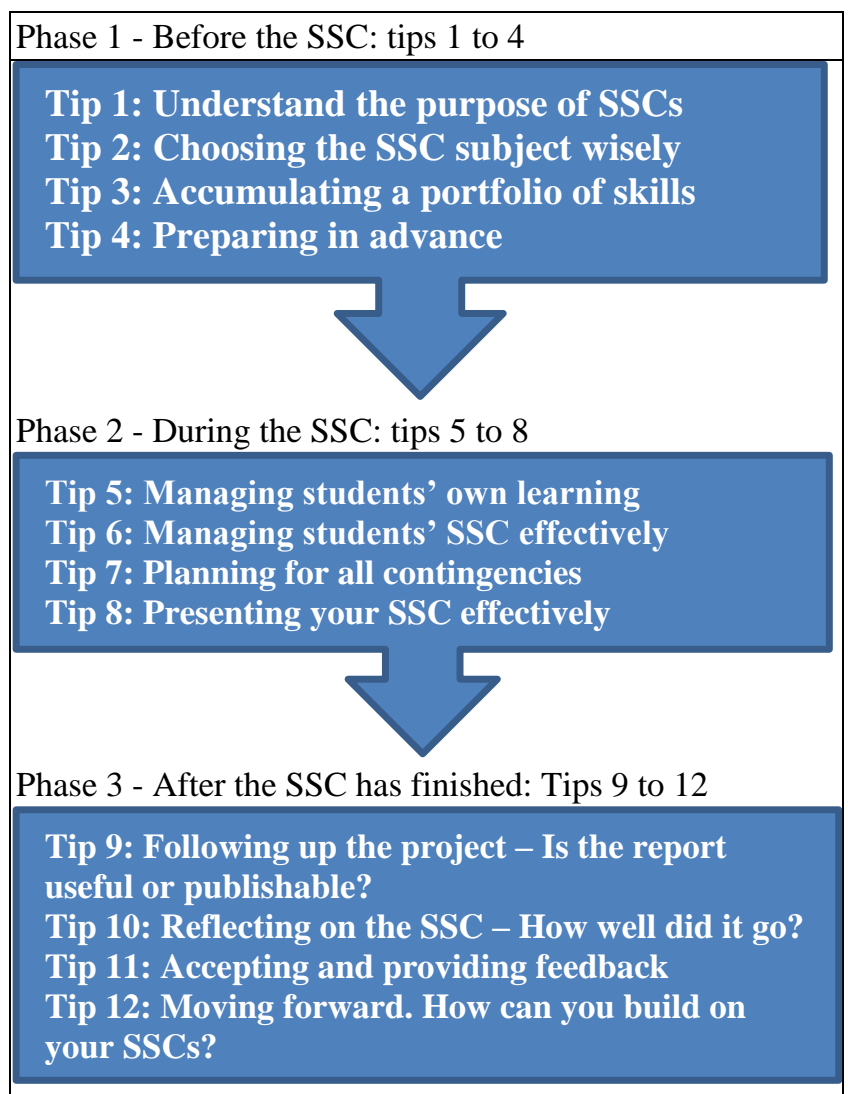

Figure 1: A three phase depiction of the 12 tips approach to undertaking SSC projects (adapted from Riley 2009). ${ }^{5}$

In the author's institution, the duration of an SSC (in year $1 \mathrm{MBChB})$ is ten weeks. It is important to highlight that this ten-week period is not solely dedicated to the SSC. Students continue to engage with other timetabled educational activities (e.g. lectures and tutorials linked to their current placements) which run in tandem with the SSC. However, students are granted time off (e.g., a halfday or whole day off per week) from their postings to focus upon SSC activities.

In this illustration, the physical base for this SSC was the Royal Infirmary of Edinburgh (RIE), Scotland. For situational context, central Edinburgh has two large teaching hospitals, the RIE and the Western General Hospital (WGH). A further teaching hospital, St. John's Hospital, is based in Livingston, a large town situated $\sim 15$ miles west of central Edinburgh. All three ('central') training institutions are closely affiliated with the University of Edinburgh Medical School. In addition, there are many other hospitals situated further afield ('peripheral') from central Edinburgh that host the education and training of medical students in the senior years. 
The three aforementioned central sites offer the equivalent of combined secondary and tertiary level hospital services for the clinical speciality of medicine of the elderly (MoE). At the time of the project, the RIE was the author's primary site of clinical practice in MoE. The RIE site accommodated three geographically distinct acute MoE wards, with a flexible bed base for 98 patients. Therefore, given the author's intent of supervising the SSC project, it made pragmatic sense to adopt the RIE as the physical base for this SSC. A further advantage was the RIE's easier accessibility for this cohort of 'pre-clinical' (year 1 MBChB) students.

The topic context for this SSC was the theme of CPT. More specifically, this project reviewed the anticholinergic burden (ACB) of medications prescribed to an inpatient cohort in a MoE service of a large ( $>900$ beds) teaching hospital (RIE). ${ }^{12-16}$

\section{RESULTS}

\section{Before the SSC: tips 1 to 4}

\section{Tip 1: understand the purpose of SSCs}

In the author's institution, the medical school bears responsibility for defining (and updating) the structure, learning objectives and coordination of student allocations to SSC groups. In practice, these tasks are made more effective by formally appointed SSC module organisers (MOs). The MOs in turn function more effectively by having access to administrative support via the medical school. In the author's institution, SSCs are delivered across both early and senior years of the MBChB curriculum. Consequently, there are named MOs for each year of training that offers SSC experience. MOs may be clinical or non-clinical senior staff members. Regardless of background, MOs should have an expressed interest in undergraduate medical teaching and training. A committee of MOs (or equivalent group) from the various $\mathrm{MBChB}$ years liaise periodically to define, appraise, and update the objectives and outcomes of SSCs. Guided by regular and systemically collated feedback (from medical students as well as STTFs), MOs work individually and collectively to plan, design and support SSC modules. Collaborative working encourages individual SSC years to complement one another. Joint MO working also allows for SSC redesigns that strategically facilitate the incremental acquisition of skills as medical students' progress from early/junior (e.g., group-projects) to senior (e.g., more individualised projects) years. Another important role of MOs and their administrative teams is the proactive identification and maintenance of a regularly updated database of key contacts and volunteer STTFs willing to host locally supported SSCs. The accepting consultant/supervisor (or other locally defined alternative tiers/ranks), assumes responsibility for indirect senior supervision of the student group. The consultant/supervisor identifies motivated, volunteer or delegated post-basic qualification doctors (ideally affiliated to the STTFs' service, for logistic ease) to offer direct supervision to student groups. In turn, the post-basic qualification doctor (s) (in either formal postgraduate training or non-training grades or roles), bear responsibility for convening SSC group meetings. They also coordinate and facilitate the day-to-day SSC group activities. The author's experience is that for STTFs to be effective, they require prior access to the learning objectives and learning outcomes of the SSC period. This is essential as the learning objectives and outcomes differ dependent upon the medical students' year of training. In the author's institution, this information was made accessible as a clearly written course/study guide provided alongside other supportive course material. For e.g., the latter included: SSC assessment modalities, key dates, names and email addresses of key contacts, ethical assessment and permission forms, guidance for producing outputs like oral presentations or posters or research abstracts, etc. The latter information is normally communicated via email to consultant/supervisors well in advance of the start date of any upcoming SSC period.

\section{Tip 2: choosing the SSC subject wisely}

In this illustration, the SSC subject was chosen in a considered and sequential manner - as detailed earlier in the methods section. The SSC was selected to be in the supervisor's primary clinical speciality of MoE. The project was then narrowed down to focus on a QIA. Thereafter, the theme of CPT was selected - it being one of the current programme themes of the Edinburgh Medical School. Once CPT was chosen, the subthemes of prescribing and polypharmacy were nominated.

Subsequently, the supervisor proposed a specific topic on: 'A review of the anticholinergic burden (ACB) of medications prescribed to an inpatient cohort in three acute MoE wards in a teaching hospital.' In proposing a final SSC topic, the supervisor found it helpful to adopt the SMART-approach to setting goals or objectives. The acronym standing for S-specific; M-measurable; Aachievable; R-relevant; and T-time-bound. In this SSC, the project topic was proposed by the supervisor. However, STTFs should note that medical students (especially in senior years) may capably identify potential SSC topic areas. This should be encouraged, as it serves as a further opportunity to promote students' personal and professional development (PPD).

For student-suggested topics, the supervisor's role could be modified towards mentorship. A mentor can then experientially guide students to refine their suggestion into a SMART-compatible project that also fits into the SSC aims and timescale. Such mentorship and role-modelling offers students the added benefits of access to a senior adviser who supports them as a strategist. An effective supervisor or mentor should seek to challenge the students in a supported manner, and nudge them encouragingly further along their professional learning curve. 


\section{Tip 3: accumulating a portfolio of skills}

In a broad sense, it was envisioned that this SSC would support medical students in completing a QI group project that reviewed the per-patient cumulative ACB of medications prescribed to a cohort of MoE inpatients in a teaching hospital. The project was designed to: serve as an experiential platform from which early-year students could learn transferable skills (e.g. team-working, timemanagement, problem-solving, data collection and interpretation, information management and governance, and basic QIA methodology).

The project was also designed to offer an experience of how specialisms (in this case CPT and MoE) could be integrated into an SSC.

To encourage students to develop further PPD skills, this SSC was designed to accommodate 'direct' supervision by a named postgraduate doctor, with access to 'indirect' supervision of a named consultant physician (the author). For e.g., to achieve this goal initial consideration was given to the medical students undertaking one of the following SSC options: 1) a detailed literature review of a topic or subtheme of CPT 2) the design and delivery of a teaching session on a topic within CPT 3) a clinical audit within the defined theme of CPT 4) a QIA project addressing a subdomain of CPT.

A final determination was made to follow through on a QIA methodology. A QIA had potential advantages of allowing the clinical service to evaluate the study findings, and to make incremental and measurable changes to clinicians' practice. The latter could in turn feed into polypharmacy reviews of older hospital inpatients, as inappropriate polypharmacy is itself an identified surrogate marker for frailty, falls, cognitive impairment and delirium.

\section{Tip 4: preparing in advance}

Preparing and planning ahead for the SSC was a crucial period, and requiring investment of time. For e.g. this involved the points listed below.

The completion of a hospital QI project registration form. Although not applicable to this project, supervisors need to judge if the proposed SSC would impact directly upon patient care. If so, formal applications for ethical approval should be completed well in advance of SSC timescales.

This phase was also an opportunity to remind students (in advance of SSC commencement) of the project topic, remit, learning objectives and outcomes. Such crucial information can support advance reading around the topic/subtheme, so that students feel more empowered to 'hit the ground running.'

As described earlier under 'methods', the supervisor had determined the hospital base for this proposed SSC. This decision was communicated to students in advance via email. Additional information relayed to students included: initial reporting arrangements - date, venue and time; details of relevant contacts e.g. the supervisors (named consultant physicians in MoE) and facilitating doctors (named post-graduate doctors/clinical fellows in $\mathrm{MoE})$.

Once a site or base is determined, notification should also be given to other key personnel within that service. This ensures that students' arrival on the wards is anticipated. For e.g., this may include notifying the departmental head or clinical director, and/or the QIA leads (or clinical audit leads, or undergraduate teaching leads - if applicable). It is also advisable to share information with relevant members of the multi-disciplinary team (MDT) whom students might encounter on the wards or during the SSC e.g., other consultant colleagues, postgraduate doctors, clinical pharmacists, senior nurses in charge of the wards, etc.

Pre-booking of dates, time, and rooms/venues was important for regular meetings of the SSC group. Touchbase meetings with STTFs were scheduled at weekly intervals. These incorporated both in-person and remote (email/telephone) discussions. As a minimum, STTFs should aim to arrange a minimum of three meetings: initial/induction, mid-project review, and end of project meeting/debriefing.

The initial/induction meeting was an opportunity to complete a 'process map' i.e., itemising the process of the proposed QIA, and considering interventions to improve the methodology.

The initial/induction meeting also provided an occasion to complete a quick 'effort/impact' matrix. The latter is another decision-making tool that can enhance QIAs, by supporting teams to more readily identify which aspects of the SSC project they would be better placed to expend their time and resources.

A belated learning point from this project was that our prearrival email communication with the students could have made advance enquiries on whether or not they would need IT equipment (e.g. personal computers, etc). For this SSC, the host department faced the challenge of providing IT facilities to students at short notice.

Equally, STTFs should plan ahead for known periods of non-availability (e.g. annual/study leave; on-call shifts etc.) so that they can coordinate alternative arrangements for supervision/facilitation if significant absences coincide with the SSC period.

Dependent upon the SSC, it may be helpful if STTFs prepare and/or send out any documentation students might find helpful (e.g. samples of blank data collection sheets, or signposting links to informative websites). For this project, the QI lead in the author's service emailed 
information out to the students (blank QI documentation, and links to QI websites).

\section{During the SSC: tips 5 to 8}

\section{Tip 5: managing students' own learning}

The medical students' access to 'direct' and 'indirect' supervision has been expatiated above in 'tip 3: accumulating a portfolio of skills.'

Beyond that, students should be highly encouraged and supported to manage their own SSC learning. This approach recognises the requirement for medical doctors in training to acquire generic and transferrable skills e.g. becoming self-directed learners who can take the initiative for their PPD.

To enhance communication within this SSC group (students and STTFs), it was also helpful for the students to nominate a lead student. This named student then assumed a role of sending a short email summary on relevant weekly updates. For e.g., the updates could summarise two to three team learning points (for that week); identify the coming week's tasks; note the team member (s) designated to complete specific tasks or aspects of tasks; as well as stating clear timescales for outstanding tasks.

In line with the ethos of professionalism and accountability, individual students then bear responsibility for communicating outcomes of their assigned tasks to the student-lead, and in a time-conscientious manner.

If the SSC works well, then inherent to these activities are crucial transferrable skills (e.g., some skills as identified earlier in 'tip 3: accumulating a portfolio of skills'). In addition, students learn more about negotiation, responsible and appropriate delegation, plus verbal and written communication. Dependent upon individual student's perceptiveness, STTFs may need to more explicitly emphasise these skills to students - as some may not innately recognise or appreciate that they have developed further (in real-time) upon these crucial skills.

\section{Tip 6: managing students' SSC effectively}

As the project unfolds, students and STTFs should maintain the tempo of actively managing the selected project.

For e.g. during this QI-related SSC on CPT, the weekly meetings and email communication served as a strategic occasion to appraise the evolving project.

The weekly communication proved helpful for highlighting important deadlines. For e.g. a planned outcome of the ACB review was the design and production of a poster. Deadlines for data summation, draft of poster write-up, timescales for editing and proofreading, and timing of group practise of oral presentations were highlighted as the project evolved. Students normally present this collectively at a formal SSC poster assessment session coordinated centrally by the medical school.

Although students are encouraged to manage the various steps in the project execution (e.g. data collation, data summation, etc.), the STTFs should be accessible to offer tips on refining strategy. In this illustration, and being a QI project on ACB of prescriptions, students were encouraged to design an initial data collection tool. Following a brief test run of the tool (collecting data from a few prescription charts), the facilitator then supported students in undertaking an early refinement of the tool. From this approach, students learned practical lessons such as: avoiding collection of redundant data; avoiding duplication during data collection; designing data collection tools in a structured format e.g. quantitative versus qualitative data, etc.

Weekly updates may also flag up students who habitually miss meetings and/or deadlines. STTFs may need to explore reasons behind why students are lagging behind their peers. To avoid alienating students further, this should be approached sensitively, preferably in confidence and away from the group. Examples of valid reasons may be: limitations in accessing IT equipment, financial constraints, disability e.g. dyslexia, recent bereavement, etc. Such issues may rightly require modified approaches to student contributions. That said, it remains crucial that the STTFs continue efforts to maintain the progress of the team's project.

Furthermore, students within an allocated SSC group may encounter conflicts of team dynamics or personality clashes. The author's view is that in some exceptional scenarios, re-allocation may be required. However, STTFs need to determine if these are individually justifiable. Beyond such case-by-case exceptions, there is an argument for medical students to be encouraged to work collaboratively and professionally with one another. For e.g., a similar professional ethos would be expected of qualified medical doctors putting aside personal differences and working for the overall benefit of a patient that they may be required to jointly care for in a real-life context.

\section{Tip 7: planning for all contingencies}

As with most projects, SSCs have no exemption from unanticipated delays or impedances.

The effective use of 'tip 4: preparing in advance,' may assist students and STTFs in identifying potential bottlenecks that may be encountered during projects. As described earlier, the completion of a 'process map,' and/or an 'effort/impact matrix' can both serve as forms of 'risk assessments' for SSCs. 
Challenges or problems may be minor (modifiable or remediable), or major (non-modifiable or non-remediable) - when considered within the timescales of an SSC.

For this illustration, we anticipated that given the ten-week limit of the SSC period, students would be unable to undertake multiple cycles of 'PDSA' - plan, do, study, act (which might form part of a larger QI process).

We also recognised that week one would be largely taken up by designing and refining the ACB medication data collection tool.

We further recognised that the last two weeks would probably be best reserved for data collation, interpretation, and the design plus production of a poster to the standard expected of a scientific presentation.

We also envisaged the possibility that data collection might be impeded by factors such as: the medical students' availability on only one day per week (Wednesdays in our case); the fact that ACB-related data was to be collected prospectively on a dynamically changing cohort of inpatients in a busy acute MoE service; and that the aforementioned issues might impact upon the sample size for the QI-based SSC. Consequently, the pragmatic decision was to design a descriptive and observational project/study, and within which no formal interventions would take place.

It is vital that planning for such contingencies is communicated to the group - before or at the initial/induction meeting.

\section{Tip 8: presenting your SSC effectively}

In this instance, students presented their ACB-based SSC project as a poster via a formal session organised by the Medical School.

This drew upon students' writing and referencing skills (the latter may vary based on the prescribed format for each school).

It was also a fitting time to reiterate to students the professional need to avoid plagiarism (including selfplagiarism), and how to appropriately cite the work of others.

Practise sessions allowed students to rehearse their oral presentation skills, and prepared them for professional critiques of their poster presentation.

\section{After the SSC has finished: tips 9 to 12}

Tip 9: following up the project - is the report useful or publishable?
This phase offers an opportunity to imbibe into students the rewards of self-attainment, but also a measure of realism about what they have accomplished.

Some projects will not be suitable for progression beyond what has been achieved so far. Others may be worthy of additional posters or oral presentations (e.g. at external meetings - regional or national). Some may even be suitable for publication (e.g. in peer reviewed journals online or paper formats).

Senior supervisors may be in a position to draw upon their experience to guide students accordingly.

\section{Tip 10: reflecting on the SSC-how well did it go?}

This is an opportunity for students and STTFs alike to reflect upon their experience of the whole project, and to consider questions like: whether they want to undertake more projects, and how experiences gained from the project could offer practical lessons for future SSCs.

In this case, students were required to award themselves 'self-assessments' and 'team-based feedback.' The focused upon a self-appraisal of how they and their teams performed during the project. These self-assessments are intended for formative purposes, and hoping to promote the development of insight into team-working and teamdynamics. Potential value is derived when students later correlate their self-assessments with the external assessments or grades awarded by a medical school appointed panel of poster assessors using pre-defined descriptors.

For example, in the author's institution, the scored domains for this SSC were: preparation for the session, participation during session, group working skills, communication skills, professional behaviour, and critical thinking.

The corresponding grade descriptors in use at the medical school at the time of this illustrated SSC were: $A=90$ $100 \%=$ excellent, $\mathrm{B}=80-89 \%=$ very good, $\mathrm{C}=70$ $79 \%=$ good, $\mathrm{D}=60-69 \%=$ pass, $\mathrm{E}=50-59 \%=$ marginal fail, and $\mathrm{F}=0-49 \%=$ clear fail

It has to be noted that alternative rating scales may be used in line with an individual medical school's marking criteria and grading scales.

\section{Tip 11: accepting and providing feedback}

This phase allows for focusing on feedback related to all SSCs undertaken within a defined period. Ideally, feedback should be: invited to make constructive suggestions for how MOs could potentially make improvements to the SSC programme(s), encouraged from students and STTFs, and centrally collated, recognised and acted upon. 
Tip 12: moving forward - how can you build on your SSCs?

The last tip involves participants (especially students) considering if they have gained better appreciation for some of their personal and professional skills.

In the author's institution, medical students normally gain experience of the speciality of MoE during their final year of the curriculum.

Therefore, this SSC offered an 'early taster' of the clinical speciality of MoE to students during year one of the sixyear MBChB programme. They also experienced an aspect of CPT, and gained non-clinical experience by undertaking a small group QI project.

One cannot directly infer the impact this experience may have upon students' future careers or interests. However, the illustrated SSC granted 'early-years' medical students educational experiences and training that went beyond their immediate placements, and complemented their other timetabled academic activities. Role-modelling is acknowledged to be of positive impact in medical education and training, and this may extend to a potential inspiration upon students' career aspirations.

\section{DISCUSSION}

Medical schools across the world offer a broad range of undergraduate curricula. Some have been described as traditional, while others have evolved and adopted alternative or innovative curricula that seek to negotiate around the potential risks of stagnation. ${ }^{1,2,17}$ One driver for curricula change is the need to better prepare future clinicians for capability and competence in problemsolving, thereby aiming to adapt service provision in alignment with the health needs of societies they serve. ${ }^{17}$

Even in medical schools that retain a more traditional split of 'pre-clinical' and 'clinical' years, SSCs may be strategically designed to run across different years of undergraduate medical education, training, feedback and assessments. ${ }^{5-9}$ This is possible because SSCs can be designed to complement or replace some existing curricula teaching and experiences in a manner that integrates into basic clinical sciences and/or clinical specialties. ${ }^{18,19}$

Further developing upon this initial broad framework, SSCs could potentially be designed to offer variation across other domains. Such opportunities may arise from clinical and non-clinical aspects of audit; QIA and methodology; quality control, management and assurance processes relating to medical education; learning experiences linked to components of research, critical review and appraisal of literature; development of medical portfolios; teaching practice; and other medical education related activities (e.g. near peer learning). ${ }^{5,6,20-23}$ Some medical schools offer an extra intercalated degree year which may offer additional research-based emphasis across their SSCs.
In this article, the SSC group project was based on a QIA chosen from the speciality of MoE and integrated with a CPT-subtopic on ACB of inpatient prescriptions. ${ }^{12-16} \mathrm{~A}$ further strategy allows for SSCs in the earlier medical undergraduate years to evolve from group-based projects and assignments, towards increasingly individualised topics as students transition into senior undergraduate years. ${ }^{3-6}$

\section{CONCLUSION}

This report illustrates how a previously described 12-tips approach to developing an SSC was employed practically to design and complete a QI project focusing on a topic of interest to CPT. The report describes the review of ACB of medications prescribed to a MoE inpatient cohort based in a large teaching hospital.

Guided by experience of the process, the article's learning points are shared in a sequential manner that allows both non-clinical and clinical medical educationalists to consider how they might design practical SSC-type projects for the training of medical students in centres that they have affiliations with.

Funding: No funding sources

Conflict of interest: None declared

Ethical approval: The study was approved by the Institutional Ethics Committee

\section{REFERENCES}

1. Murdoch-Eaton D, Ellershaw J, Garden A. Studentselected components in the undergraduate medical curriculum: a multi-institutional consensus on purpose. Med Teacher. 2004;26:33-8.

2. Payne G, Thomson A, Flood C. Special study modules should be more diverse. Student $\mathrm{Br}$ Med J. 2000;8:468.

3. Bidwai A. SSMs are my saviour. Student Br Med J. 2000;8:339-40.

4. Cross P. Getting the most out of SSMs. Student Br Med J. 2003;11;336-7.

5. Riley, SC. Ferrell, WR, Gibbs, TJ, Murphy, MJ and Smith, WCS. Twelve tips for developing and sustaining a programme of student selected components. Med Teacher. 2008;30:370-6.

6. Riley SC, Gibbs TJ, Ferrell WR, Nelson PR, Smith WCS, Murphy MJ. Getting the most out of student selected components: 12 tips for participating students. Med Teacher. 2009;31(10):895-902.

7. Riley SC. Student Selected Components: AMEE Guide 46. Association for Medical Education in Europe (AMEE), Dundee, UK. 2010.

8. Murphy MJ, Seneviratne RDA, Remers OJ, Davis MH. Student selected components: student-designed modules are associated with closer alignment of planned and learnt outcomes. Med Teacher. 2009;31:489-93. 
9. Riley SC. Student Selected Components: AMEE Guide 46. Med Teacher. 2009;31:885-94.

10. Hounsell D, McCune V, Hounsell J, Litjens J. The quality of guidance and feedback to students. Higher Educ Res Develop. 2008;27:55-67.

11. Murphy MJ, Seneviratne RDA, McAleer, SP, Remers OJ, Davis MH. Student selected components: do students learn what teachers think they teach? Med Teacher. 2008;30:175-9.

12. Gannon E, Mallon E, Lannon R. 206 Audit of Anticholinergic Medication Burden in Nursing Home Residents and Associated Falls Risk. Age Ageing. 2018;47(5):13-60.

13. Marcum Z, Wirtz H, Pettinger M, LaCroix A, Carnahan R, Cauley J, et al. Anticholinergic medication use and falls in postmenopausal women: findings from the women's health initiative cohort study. BMC Geriatr. 2016;16(1).

14. Zia A, Kamaruzzaman S, Myint $P$, Tan $M$. Anticholinergic burden is associated with recurrent and injurious falls in older individuals. Maturitas. 2016;84:32-7.

15. Fox C, Richardson K, Maidment I, Savva G, Matthews F, Smithard D, et al. Anticholinergic Medication Use and Cognitive Impairment in the Older Population: The Medical Research Council Cognitive Function and Ageing Study. J Am Geriatric Soc. 2011;59(8):1477-83.

16. Aizenberg D, Sigler M, Weizman A, Barak Y. Anticholinergic Burden and the Risk of Falls Among
Elderly Psychiatric Inpatients: A 4-Year Case-Control Study. Int Psychogeriatr. 2002;14(3):307-10.

17. Ogundipe OA. Undergraduate training in geriatric medicine in the UK. Age Ageing. 2007;36(1):109-10.

18. Newbegin RM, Rhodes JC, Flood LM, Richardson HC. Student-selected components: bringing more ENT into the undergraduate curriculum. J Laryngol Otol. 2007;121:783-5.

19. Fletcher G, Agius RM. The Special Study Module: a novel approach to undergraduate teaching in occupational medicine. Occup Med. 1995;45:326-8.

20. Ogundipe OA. Knowledge-based assessments: maintaining rigour in standard setting processes. Clin Med. 2007;7(2):200-1.

21. Qureshi S, Jones H, Adamson J, Ogundipe OA. Ageing Simulation for Promoting Empathy in Medical Students. BMJ Simulation \& Technology Enhanced Learning. BMJ Stel. 2017;3(2):79-81.

22. Ogundipe OA. Education and Training Update: Maintaining an Effective Medical Training Portfolio. Brit J Hosp Med. 2008;69(10):587-9.

23. Furmedge DS. Teaching skills: a school-based special study module. Med Educ. 2008;42:1140.

Cite this article as: Ogundipe OA. Student selected components as an educational platform for teaching medical students about clinical pharmacology and quality improvement activities. Int J Basic Clin Pharmacol 2021;10:309-16. 